\title{
La forza delle lingue deboli; la debolezza delle lingue forti
}

\section{Luisa Revelli}

\section{(2) OpenEdition \\ 1 Journals}

Edizione digitale

URL: http://journals.openedition.org/esp/906

DOI: $10.4000 /$ esp.906

ISSN: 2532-0319

\section{Editore}

Centre d'Information sur l'Éducation Bilingue et Plurilingue

\section{Edizione cartacea}

Data di pubblicazione: 1 dicembre 2016

Paginazione: 5-12

ISSN: 1127-266X

\section{Notizia bibliografica digitale}

Luisa Revelli, « La forza delle lingue deboli; la debolezza delle lingue forti », Éducation et sociétés

plurilingues [Online], 41 | 2016, Messo online il 20 octobre 2017, consultato il 24 avril 2019. URL : http:// journals.openedition.org/esp/906 ; DOI : 10.4000/esp.906 


\section{LA FORZA DELLE LINGUE DEBOLI; LA DEBOLEZZA DELLE LINGUE FORTI*}

\section{Luisa REVELII}

Conçue comme cadrage aux deux journées consacrées aux «langues faibles» organisées conjointement par le CIEBP et par l'Université de la Vallée d'Aoste (Aoste, 28-29 Octobre 2016), la contribution vise à fournir quelques réflexions pour la discussion des concepts de langue faible - langue forte, qui, selon la perspective adoptée, peuvent exprimer des significations très différentes et même antithétiques. Dans le but de mettre en évidence la valeur relative des deux locutions, sont proposées des réflexions sur le rôle joué par les représentations individuels qui, formulées sur la base d'évaluations culturelles, identitaires, idéologiques, politiques, économiques mais aussi esthétiques et émotionnelles, peuvent influencer les options de changement, alternance, conservation et transmission intergénérationnelle des langues de famille, les choix de récupérer des langues d'onigine, les attitudes adoptées dans les étapes d'apprentissage des langues maternelles et étrangères et, plus généralement, peuvent modifier les perceptions individuelles et collectives de force ou de faiblesse des langues proches et lointaines, les siennes propres et celles des autres.

Mots-clés: terminologie du multilinguisme, représentations des langues, sentiment linguistique, transmission intergénérationnelle des langues, phonesthétique

Introducing the Conference "Weak languages» organized jointly by the CIEBP and the University of the Aosta Valley (28-29 October 2016), this article discusses the concepts of weak and strong languages, which, depending on the point of viewe, can have very different and even opposite meanings. To highlight the relative values of the two expressions, we offer some suggestions as to the role played by individual representations which, based on cultural, identity-linked, ideological, political and economic, as well as aesthetic and emotional evaluations, can impact the decisions to change, alternate, conserve or transmit the family language(s) from one generation to the next, the return to a language of origin, the attitudes to language learning and, more generally, the individual and collective perceptions of the strength or weakness of languages, familiar or foreign, one's own or other people's.

Key-words: terminology of multilingualism, representations of languages, linguistic sense, transmission of languages between generations, phono-aesthetics

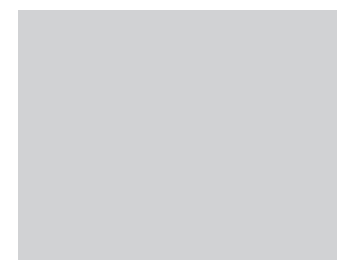

I n qualsiasi ambito e probabilmente in qualunque idioma le nozioni di forza e debolezza rimandano a concetti antitetici che ne rendono inopportuno l'accostamento, se non con deliberati intenti ossimorici. Quando però gli attributi di forza e debolezza si riferiscano alle lingue, come nel nostro titolo, l'effetto contrappositivo non costituisce una scelta stilistica, ma risponde invece a un tentativo di condensata messa in rilievo 
La forza delle lingue deboli; la debolezza delle lingue forti L. ReVeluI delle criticità terminologiche esistenti per la descrizione di un tema che pur avendo conquistato uno spazio significativo in ambiti d'indagine di differenti settori della ricerca, e anzi forse proprio per l'eterogeneità delle prospettive da cui può essere osservato - tende ad essere descritto, non solo in italiano, attraverso locuzioni che possono risultare ambigue o anche fuorvianti (1). Ciò che si debba intendere per lingua forte e lingua debole, in effetti, può variare in modo molto significativo a seconda di scopi, circostanze, contesti.

Nell'opinione comune, un po' grossolanamente ma in genere in modo condiviso, una lingua viene qualificata come forte (o dominante) sulla base di parametri fondamentalmente politico-economici e sociali (numero di parlanti che la utilizzano, ampiezza degli spazi in cui viene impiegata, riconoscimenti ufficiali e valori simbolici di cui gode, potere commerciale, ecc.), mentre l'espressione lingue deboli è specularmente associata ai repertori comunitari caratteristici dei contesti di plurilinguismo endogeno: la locuzione, in sostanza, allude alle cosiddette minoranze linguistiche di antico insediamento, potenzialmente esposte a rischi di progressiva obsolescenza (indebolimento), e occorre quindi spesso in riferimento a questioni di politica linguistica locale, nazionale e sovranazionale.

In un'ottica ecolinguistica (Valentini et al. 2003) il concetto di lingua debole si definisce più specificamente in rapporto al contesto socio-ambientale in cui gli idiomi sono collocati e prevede la possibilità di intercettare eventuali segni di regressione o declino all'interno del repertorio attraverso indicatori di vitalità che fanno normalmente riferimento a fattori esterni (cfr. Moretti et alii. 2011, e in particolare le Considerazioni conclusive di Gaetano Berruto alle pp. 289-302), relativi alla comunità parlante nel suo complesso, potendo prendere in considerazione anche parametri quali i livelli di competenza ed eventualmente dati qualitativi sulle rappresentazioni individuali del repertorio di riferimento, ma sempre ai fini di un'analisi collettiva, piuttosto che riferita ai singoli parlanti.

Dal punto di vista di questi ultimi, soprattutto nelle prospettive di taglio psicolinguistico e glottodidattico, l'attributo della forza può invece essere assegnato anche a lingue comunemente rappresentate come deboli ma compiutamente possedute dal parlante, come nel caso di una parlata locale acquisita come lingua materna e utilizzata con continuità in ambiti diversificati. Il concetto della debolezza, per converso, può rimandare a situazioni di competenza deficitaria, transitoria o incompleta tipiche anche delle varietà di apprendimento formale di lingue dallo statuto forte, oppure a casi di uso circoscritto di lingue ufficiali ma laterali negli usi, di abilità frammentarie nelle varietà native dei genitori, di competenze disponibili ma latenti in parlanti evanescenti (2).

Possono d'altra parte essere definite deboli, sia in una prospettiva di linguistica interna che di linguistica esterna, anche le lingue migranti e ciò pure 
La forza delle lingue deboli; la debolezza delle lingue forti

L. ReVeluI quando siano caratterizzate di partenza da uno statuto di lingue forti (perché sancite come lingue 'ufficiali' o 'nazionali') ma, trapiantate in contesti in cui l'uso rimane costretto a livello di comunità o famiglia, siano in tali ambiti soggette all'erosione dei codici indigeni e quindi esposte al rischio dell'abbandono.

A ben vedere, in effetti, qualunque codice - sia esso endogeno o esogeno, acquisito come lingua materna o in apprendimenti successivi - può manifestarsi come forte o debole a seconda del ruolo che il parlante, più o meno consapevolmente e intenzionalmente, stabilisce di attribuirgli. Una lingua seconda o straniera può, anche se formalmente forte, risultare debole tutte le volte in cui, in un bilancio sommativo, $\mathrm{i}$ benefici derivanti da un suo uso stabile e continuativo siano percepiti come inferiori agli sforzi richiesti per renderla o mantenerla attiva e vitale; quando, piuttosto che spontaneamente acquisita senza fatica e fluentemente processata, comporti compiti di elaborazione onerosi e poco motivanti; quando sia sistematicamente sottoposta alla concorrenza di un codice alternativo e rispetto ad esso sia avvertita come economicamente, culturalmente, affettivamente subalterna; quando occupi uno spazio emotivo angusto o conflittuale, e quindi il parlante la percepisca come distante.

Una lingua debole in origine può, per contro, essere avvertita come forte per la sua capacità di favorire il mantenimento o anche la costruzione di un'identità etnica, di incarnare la memoria e continuità affettiva di una storia o tradizione, di costituire l'anello di socializzazione e integrazione nell'ambito di una comunità coesa, di essere il collegamento più tangibile con quella di partenza, se geograficamente distante; di rimandare simbolicamente a universi fortemente evocativi e pregnanti, decisivi dell'aggregazione interna fra alcune o tutte le generazioni dei membri di un nucleo familiare; può infine, anche se formalmente debole, semplicemente configurarsi come forte rispetto alle competenze, agli usi, alle rappresentazioni estetiche e ai giudizi valutativi individuali, nella misura in cui, secondo l'enunciazione terraciniana, "il sentimento della propria lingua conduce il parlante a sentirla distinta da ogni altra, come l'unica lingua, la lingua per eccellenza" (Terracini 1957, p. 207).

Ed è probabilmente proprio la categoria del sentimento linguistico nelle sue molteplici componenti - culturali, identitarie, ideologiche, estetiche, affettive ed altro ancora - a rendere ardue le interpretazioni e le previsioni a proposito degli andamenti relativi ai repertori di famiglie e comunità: dispositivi relativi a fenomeni quali la trasmissione intergenerazionale, chiave decisiva della conservazione delle lingue su piccola e larga scala, risultano infatti sfuggenti per la numerosità e indefinibilità delle variabili emotive coinvolte tanto alle misurazioni quantitative quanto alle valutazioni qualitative (3). Dietro alla decisione di tramandare alle generazioni successive un codice familiare potenzialmente debole possono infatti convergere sen- 
La forza delle lingue deboli; la debolezza delle lingue forti

L. ReVeluI timenti ambivalenti e spinte divergenti, che rimandano, da un lato e per esempio, a una volontà di conservazione della propria identità etnico-culturale di matrice ostile verso il contesto 'altro', maggioritario o d'arrivo; che possono sottendere, all'opposto, una matura coscienza del plunilinguismo (Candelier et al. 2008) fondata sulla disponibilità a una più ricca seppur antieconomica gestione delle relazioni comunicative. L'indubitabile volontà di conservazione di un codice manifestata dalla trasmissione intergenerazionale, insomma, può costituire il risultato di sentimenti contrari, soggetta com'è a rappresentazioni emotive ed emotivamente manipolabili.

Gli ultimi due secoli di storia italiana ed europea possono, in questo senso, fornire esempi molto concreti: alle credenze sulla nocivité du bilinguisme alimentate tra Otto- e Novecento per contrastare l'uso di codici ritenuti antagonisti delle lingue standard, nazionali e ufficiali (TabouretKeller 2011) si è sostituita, agli albori del XXI secolo, una generale e spesso ingenuamente fervida rappresentazione dei vantaggi individuali, sociali e anche economici della possibilità di governare più lingue contemporaneamente. Principalmente ma non soltanto in ambito educativo, una dilagante sottovalutazione del carico cognitivo cui un parlante è di fatto esposto nelle fasi di apprendimento formale di più codici e nella processazione dei contenuti disciplinari eventualmente da questi veicolati tende oggi a diffondere una visione secondo la quale la sommaria conoscenza di più lingue dovrebbe occupare una posizione prevalente sulla conservazione del codice (endogeno o esogeno) della socializzazione primaria e prioritaria anche rispetto al raggiungimento di competenze, orali e scritte, tali da consentire lo svolgimento di compiti cognitivi di un certo spessore nella principale lingua d'uso scolastico ed extrascolastico.

Si tratta di concezioni che rientrano in quella che Rosanna Sornicola (2013: p. 57) efficacemente definisce 'mistica' del multilinguismo, precisando che "una celebrazione acritica e per così dire trionfalistica del multilinguismo dovrebbe essere respinta, non meno dei pregiudizi sugli effetti negativi del bilinguismo eletti a generalizzazioni scientifiche" (4). Si tratta, peraltro, di ambiti di riflessione che toccano questioni didattiche a cui diversi linguisti si sono dedicati con l'obiettivo di offrire un contributo all'educazione linguistica e di migliorarne le prassi. Cito, fra i molti d'ambito italiano, Tullio Telmon, membro del CIEBP attivo nell'ambito dell'iniziativa Langues faibles, che accogliendo "con grande soddisfazione l'inserimento della lingua straniera nel percorso curricolare" della scuola primaria italiana ha avuto modo di segnalare in modo inequivocabile le insidie insite nell"'abuso di termini quali bilinguismo" e "i grossi rischi di insuccesso che questa innovazione comporta se la prassi didattica continua a ignorare, come fa, la differenza tra bilinguismo e diglossia" poiché "la sociolinguistica ci insegna che l'asimmetria, e dunque la diglossia e non il 
La forza delle lingue deboli; la debolezza delle lingue forti

L. ReVeluI bilinguismo, sono le sole realtà concrete in fatto di coesistenza e di contatto di più lingue in una società. Sarebbe molto saggio, molto utile e soprattutto molto proficuo che la scuola prendesse atto di ciò e riducesse i propri obiettivi in fatto di insegnamento di una lingua straniera, provando a individuare talune funzioni e taluni domini da privilegiare e da destinare alla sola L2 e limitando conseguentemente a tali funzioni e domini il proprio intervento" (Telmon 2016: 300-301).

Ma temi come questi, che chiamano in causa assunti complessi al cui interno si ingarbuglia una moltitudine di componenti sensibili e articolate, esposte a impopolari rischi di fraintendimento e banalizzazione, a valutazioni parziali, a interpretazioni strumentali, a letture utilitaristiche, a mode e interessi commerciali, raramente, anche fuori dalla realtà italiana, riescono a raggiungere efficacemente il mondo della scuola, costituito - prima ancora che da docenti e alunni - da decisori politici e amministratori.

Nascono allora anzitutto da esigenze di confronto e divulgazione scientifica le due giornate seminariali organizzate dal CIEBP in collaborazione con l'Università della Valle d'Aosta per le date del 28 e 29 ottobre 2016 con lo scopo di fornire a studenti, insegnanti e cittadini interessati all'argomento un'occasione di riflessione critica a proposito dei ruoli che famiglie, scuole e parlanti tutti possono assumere rispetto agli assetti e alle diverse componenti delle rappresentazioni di forza e di debolezza delle lingue.

Spetterà a Bruno Moretti (Università di Berna), in apertura, il compito di introdurre il tema con un contributo di taglio sociolinguistico il cui titolo - non a caso espresso con formulazione interrogativa («Lingue deboli?») - porrà le basi per una relativizzazione del concetto di debolezza con particolare riferimento ai rapporti tra dialetti e lingue nazionali e alle specificità del caso svizzero.

Seguirà un contributo di Gabrielle Varro la quale - a partire da esempi riferiti alle dinamiche interlinguistiche proprie delle famiglie miste (cfr. Varro 2003; Deprez et alii 2014) che si trovano a dover «transmettre aux enfants au prix d'énormes efforts deux langues - une minoritaire (mais forte: l'anglais, l'allemand, ...) du/de la parent(e) ?? transplanté-e - et une majoritaire (le français)» - si proporrà di mettere in evidenza «le statut relatif et variable des langues sur le plan individuel, bien qu'elles soient collectivement socialement et politiquement dominantes».

La natura contraddittoria delle dinamiche linguistiche di forza e debolezza sarà esaminata con specifico riferimento alla lingua italiana in due successivi contributi: nel primo, Silvia Natale ed Etna Krakenberger (Istituto di Lingua e Letteratura italiana dell'Università di Berna) presenteranno i risultati di un'indagine condotta a proposito delle abitudini linguistiche e delle reti sociali dei cosiddetti 'cervelli in fuga', ossia dei giovani italiani con un 
La forza delle lingue deboli; la debolezza delle lingue forti

L. ReveluI elevato grado di istruzione trasferiti per motivi di lavoro nella Svizzera tedesca; nel secondo, Simona Cannito e Gianmario Raimondi (Dipartimento di Scienze Umane e Sociali - Università della Valle d'Aosta) illustreranno gli esiti di un lavoro di tesi dedicato allo studio delle rappresentazioni dell'italiano e dell'identità italiana espresse da famiglie di migranti di prima e seconda generazione a Monaco di Baviera.

Il tema delle lingue migranti verrà poi indagato con riferimento all'età infantile e ai contesti educativi attraverso una comparazione tra le dimensione scolastica francese e quella valdostana. In tale ambito, Gabriella Vernetto, Ispettrice scolastica della Sovraintendenza agli Studi valdostana, presenterà un progetto didattico concepito con obiettivi pedagogici di inclusione scolastica ("Des parents conteurs ou comment faire entrer les langues familiales à l'école") e testato in alcune scuole della regione. Il coinvolgimento di genitori di alunni inseriti nelle classi interessate dalla sperimentazione consentirà di disporre di testimonianze dirette che contribuiranno ad arricchire la discussione in una chiave che, nel riconoscere uno spazio cruciale alle politiche linguistico-educative, prenderà in considerazione anche le correlazioni tra le dinamiche intrafamiliari della socializzazione primaria e i ruoli della dimensione emotivoaffettiva individuale nei processi di acquisizione e apprendimento delle lingue.

Vissut, percezioni, rappresentazioni, giudizi, atteggiamenti individuali e soggettivi nei confronti delle lingue diventeranno i principali oggetti d'indagine della seconda parte del seminario, collocata nella mattina successiva, il sabato 29 ottobre. I lavori saranno allora introdotti da una rassegna di testimonianze videoregistrate di parlanti, adulti e bambini, che sollecitati ad esprimere attraverso auto-osservazioni, auto-dichiarazioni e auto-valutazioni impressioni legate alle loro esperienze, giudizi fono-estetici e opinioni personali a proposito di lingue materne, seconde e straniere - forniranno esempi di riflessione metalinguistica in atto e quindi spunti di discussione a proposito di come e quanto, già in età infantile, le rappresentazioni dei parlanti sulle lingue assumano definite e talora esibite configurazioni, situandosi in posizioni di altalenante e non sempre consapevole bilico tra sentimenti di attaccamento e percezioni di alterità, senso di appartenenza e distanze valoriali, valutazioni utilitaristiche e pregiudizi, attribuzioni di stigma o prestigio ai codici del repertorio e a quelli potenzialmente disponibili.

Alla soggettività emotiva delle rappresentazioni linguistiche sarà quindi ispirata la Tavola rotonda di chiusura che, intestata allo scritto "Peut-on dire d'une langue qu'elle est belle?" del primo Presidente CIEBP, André Martinet (1965), sarà condotta in un virtuale passaggio di testimone dall'attuale Presidente dell'associazione, Andrée Tabouret-Keller, professeur émérite all'Università di Strasburgo e autrice di numerosi studi e testi 
La forza delle lingue deboli; la debolezza delle lingue forti

L. ReveluI

\section{BIBLIOGRAFIA}

dedicati al tema del plurilinguismo. Il dibattito costituirà l'occasione conclusiva per riflettere sui potenziali condizionamenti che i guudizi ingenui, formulati sulla base di valutazioni estetiche e di sentimenti linguistici 'inesorabilmente soggettivi' (5), possono attivare nelle opzioni di cambio, alternanza, conservazione e trasmissione intergenerazionale delle lingue familiari, nelle scelte di recupero delle lingue etniche e d'origine da parte di migranti di seconda e terza generazione, negli atteggiamenti adottati nelle fasi di apprendimento delle lingue materne e straniere e, più in generale e conclusivamente, nelle rappresentazioni individuali e comuni di forza o debolezza delle lingue vicine e lontane, proprie e altrui.

CANDELIER M. et al. (a cura di). 2008. Pratiques, représentations et interventions, Presses Universitaires de Rennes.

DEPREZ C., COLLET B., VARRO G. 2014. Familles plurilingues dans le monde. Mixités conjugales et transmission des langues. Langage $\mathbb{E}^{\circ}$ Société 147.

FUSCO F. 2007. Le minoranze linguistiche: una storia attraverso $i$ termimi. In: Vicini / lontani. Identità e alterità nella/della lingua, a cura di E.Pistolesi e S.Schwarze, Frankfurt am Main, Lang, pp. 89-113.

IANNACARO G. e DELL'AQUILA V. 2007. Pre-vedere il cambiamento: analisi e previsione dell'evoluzione degli usi dei codici in territori plurilingui. In: Actes du XXVe Congrès International de Linguistique et de Philologie Romane a cura di M. Iliescu et al., Berlin-New York, De Gruyter, vol. 1, pp. 173-183.

MARTINET A. 1965. Peut-on dire d'une langue qu'elle est belle? Revue d'esthétique, Nouvelle Série 3-4, XVIII, pp. 227-239.

MORETTI B. 1999. Ai margini del dialetto. Varietà in sviluppo e varietà in via di riduzione in una situazione di 'mizio di decadimento', Bellinzona, Osservatorio linguistico della Svizzera italiana.

MORETTI B., E. M. PANDOLFI, M. CASONI (a cura di) 2011. Vitalità di una lingua minoritaria. Aspetti e proposte metodologiche, Bellinzona, Osservatorio linguistico della Svizzera italiana.

TABOURET-KELLER A. 2011. Le bilinguisme en procès, cent ans d'errance (1840-1940), Limoges, éditions Lambert-Lucas.

TELMON T. 2002. Le ragioni di un titolo. In: Che cosa ne pensa oggi Chiaffredo Roux. Percorsi della dialettologia percezionale all'alba del nuovo millennio a cura di M.Cini e R.Regis, Alessandria, Edizioni dell'Orso, pp. V-XXXIV.

TELMON T. 2016. Aspetti sociolinguistici del bilinguismo. In: Pagine scelte, a cura di S.Canobbio et al., Alessandria, Edizioni dell'Orso, pp. 293301.

SORNICOLA R. 2013. Abbiamo bisogno di una linguistica delle emozioni? In: Di linguistica e sociolinguistica. Studi offerti a Norbert Dittmar, a cura di 


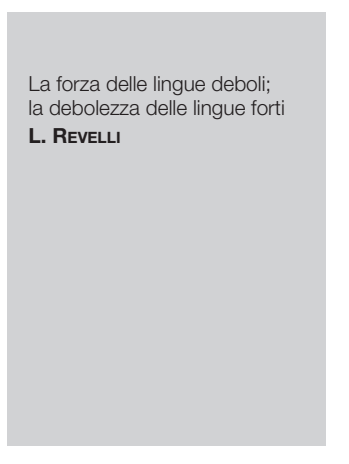

I.Tempesta, M.Vedovelli, Roma, Bulzoni, pp. 49-76.

TERRACINI B. 1957. Conflitti di lingue e di cultura, Venezia, Neri Pozza Editore.

VALENTINI A., P. MOLINELLI, P. CUZZOLIN, G. BERNINI. 2003. Ecologia linguistica. Atti del XXXVI congresso internazionale di studi della Società di Linguistica Italiana (SLI), Roma, Bulzoni.

VARRO G. 2003. Sociologie de la mixité, Paris, Editions Belin.

\section{NOTE}

* Introduzione alle Giornate di Studi 'Langues faibles', CIEBP-UniVdA, 28-29 ottobre 2016.

(1) Come osserva Fabiana Fusco (2007: p. 90) in relazione alla varietà e discontinuità della terminologia relativa ai fenomeni del plurilinguismo, "tanto la proliferazione di termini tecnici riconducibile a scuole e approcci distinti quanto la sovrapposizione di termini diversi che celano invece una sostanziale identità di opinioni sono fatti particolarmente insidiosi quando ci si deve servire di un metalinguaggio per parlare del linguaggio stesso".

(2) Ossia, secondo una semplificazione della ben più articolata definizione coniata da Bruno Moretti (1999) in rapporto al dialetto ticinese, coloro che, pur potenzialmente avendo la possibilità di farlo in quanto competenti almeno a livello ricettivo, non parlano il dialetto.

(3) Un interessante tentativo di previsione linguistica basato sulla comparazione diacronica delle dichiarazioni sulle lingue parlate dagli intervistati con persone di generazioni diverse è stata compiuta da Gabriele Iannacaro e Vittorio Dell'Aquila (2007) nell'area delle valli ladine delle Dolomiti. La metodologia adottata consente agli Autori di prefigurare concretamente futuri scenari riferiti alla dimensione quantitativa e comunitaria, ma esclude la presa in considerazione della variabile emotiva nelle scelte individuali.

(4) Precisa l'Autrice (p. 57): "Le tesi secondo cui l'esposizione a più lingue e la pratica in parallelo di queste provocherebbe danni appaiono ingenue e prive di solidità scientifica, ma una cosa è rigettarle, un'altra negare che il multilinguismo possa indurre problemi emotivi e cognitivi, oltre che sociali. Bisogna ammettere con franchezza che, accanto ad aspetti positivi, il multilinguismo comporta anche non poche difficoltà. Negare che un individuo bilingue (multilingue) e il suo contesto familiare e sociale siano spesso più esposti a difficoltà emotive e di interazione sociale equivarrebbe a chiudere gli occhi su realtà che hanno una forza e una consistenza empiricamente verificabili attraverso l'esame di situazioni storiche, geografiche e culturali diverse".

(5) Così, commentando Terracini, Tullio Telmon (2002: p. XI) definisce il 'sentimento intuitivo' del parlante per differenza rispetto alla 'sensibilità percettiva' del linguista, osservatore estraneo la cui oggettività 'è in ogni caso condizionata dal grado della sua acutezza di osservazione differenziale". 